\title{
On the Accuracy of the Gaussian Approximation for the Evaluation of Nonlinear Effects in OFDM Signals
}

\author{
Teresa Araújo and Rui Dinis
}

\begin{abstract}
The almost Gaussian nature of OFDM (Orthogonal Frequency Division Multiplexing) signals with high number of subcarriers $N$ is widely employed to characterize nonlinearly distorted OFDM signals and to evaluate the corresponding performance. In this paper we study the accuracy of the Gaussian approach when evaluating nonlinear effects in OFDM signals with finite number of subcarriers, showing the strengths and limitations of this approach.

It is shown that the decomposition in useful and selfinterference components is valid even for a reduced number of subcarriers. The Gaussian approximation of the nonlinear selfinterference at the subcarrier level is very accurate provided that $N^{2}$ is high. However, the nonlinear distortion levels slightly lower than the ones obtained with the Gaussian approximation, with relative errors dropping with $1 / N$, leading to somewhat pessimistic SIR levels (Signal to Interference Ratio).
\end{abstract}

Index Terms-OFDM signals, nonlinear distortion, Gaussian approximation, intermodulation analysis.

\section{INTRODUCTION}

$\mathbf{O}$ FDM (Orthogonal Frequency Division Multiplexing) modulations can have excellent performance in severely time-dispersive channels without the need of complex receiver implementations, making them suitable for broadband wireless systems. For this reason, they were selected for several systems, such as DVB (Digital Video Broadcasting), wireless broadband access technologies IEEE $802.16 \mathrm{a} / \mathrm{d} / \mathrm{e}$, commonly referred to as WiMAX and 3GPP (3rd Generation Partnership Project) LTE (Long Term Evolution).

However, OFDM signals have strong envelope fluctuations, making them very prone to nonlinear distortion effects [1], [2]. In fact, when the number of subcarriers is high OFDM signals have a Gaussian-like nature. This Gaussian nature can be used to characterize an OFDM signal submitted to a nonlinear device and this characterization can then be employed for performance evaluation of nonlinearly distorted OFDM signals [3]-[5]. The major outcomes that result from this Gaussian approximation are:

- Following Price's theorem [6] the nonlinearly distorted OFDM signals can be decomposed in uncorrelated useful and self-interference components;

Paper approved by C.-L. Wang, the Editor for Equalization of the IEEE Communications Society. Manuscript received March 8, 2011; revised July 7, 2011.

T. Araújo is with the Instituto de Telecomunicações, Lisboa, Portugal, and the Departamento de Matemática, Instituto Superior de Engenharia do Porto, Rua Dr. António Bernardino de Almeida, 431, Porto, Portugal (e-mail: tpa@isep.ipp.pt).

R. Dinis is with the Instituto de Telecomunicações, Lisboa, Portugal, and the Departamento de Engenharia Electrotécnica, Faculdade de Ciências e Tecnologia, Universidade Nova de Lisboa, Caparica, Portugal (e-mail: rdinis@fct.unl.pt).

Digital Object Identifier 10.1109/TCOMM.2011.102011.110151
- Using classical IMP (Inter-Modulation Product) analysis [7], we can obtain analytically the PSD (Power Spectral Density) of the self-interference component, as well as the PSD of the overall transmitted signal [5];

- The signal-to-interference ratio and signal-to-noise plus interference ratio can be obtained analytically [3]-[5];

- The nonlinear self-interference component is Gaussian at the subcarrier level, allowing an accurate computation of the BER (Bit Error Rate) performance [5].

Since practical OFDM schemes have a finite number of subcarriers, the Gaussian approximation of OFDM signals might not be accurate, especially when the number of subcarriers is not very high. An accurate characterization of the effects of nonlinear devices distortion is, therefore, critical to the future of multicarrier techniques. This paper offers such a characterization. In [8] an accurate model for the statistical characteristics of the nonlinear noise caused by Cartesian clipping of the high peak values of the in-phase/quadrature components of the baseband OFDM signal.

In this paper we present an exact characterization of OFDM signals submitted to a bandpass memoryless nonlinear device with odd nonlinear characteristics of the form $g(R)=R^{2 p+1}$. These characterizations are used to evaluate the accuracy of the Gaussian approximation for a given number of subcarriers.

This paper is organized as follows: Sec. II presents the exact characterization of nonlinearly distorted multicarrier signals and a discussion on the accuracy of the Gaussian approximation is made in Sec. III. Finally, Sec. IV is concerned with the conclusions of this paper.

\section{EXACT CHARACTERIZATION OF NONLINEARLY DiSTORTED OFDM SIGNALS}

Let us consider the transmission of a nonlinearly distorted multicarrier signal. The time-domain block $\left\{s_{n} ; n=\right.$ $\left.0,1, \ldots, N^{\prime}-1\right\}=\operatorname{IDFT}\left\{S_{k} ; k=0,1, \ldots, N^{\prime}-1\right\}$ is given by

$$
s_{n}=\frac{1}{\sqrt{N}} \sum_{k=0}^{N-1} S_{k} e^{j 2 \pi k n / N^{\prime}}=\frac{1}{\sqrt{N}} \sum_{k \in \mathcal{K}^{(1)}} S_{k} e^{j 2 \pi k n / N^{\prime}}
$$

with $\mathcal{K}^{(1)}=\{0,1, \ldots, N-1\}$. If a signal is submitted to a bandpass memoryless nonlinearity then the complex envelope of the signal at the nonlinearity output can be written as $y(t)=$ $A(R) e^{j(\arg (x(t))+\Theta(R))}$,

$$
y(t)=g(|x(t)|) e^{j \arg (x(t))}=A(R) e^{j(\arg (x(t))+\Theta(R))},
$$

with $R=R(t)=|x(t)|$ and $A(R)=|g(R)|$ and $\Theta(R)=$ $\arg (g(R))$ denoting the so-called AM-to-AM and AM-to-PM conversions, respectively [9]. We will assume an odd bandpass 
memoryless nonlinear whose characteristic can be expanded as a power series of the form

$$
g(R)=\sum_{m=0}^{+\infty} \beta_{m} R^{2 m+1},
$$

where coefficients $\beta_{m}$ are generally complex numbers. Thus the samples at the output of the bandpass memoryless nonlinearity device that operates on the oversampled version of the multicarrier signal can be written as $s_{n}^{C}=g\left(s_{n}\right)=$ $\sum_{m=0}^{+\infty} \beta_{m} s_{n}^{(2 m+1)}$, where $s_{n}^{(2 m+1)}=\left(s_{n}\right)^{m+1}\left(s_{n}^{*}\right)^{m}$.

\section{A. Nonlinear Characteristic of Order 3}

We will first focus on the case of a cubic bandpass memoryless nonlinear characteristic, i.e., $\beta_{m}=0$ for $m \geq 2$. Without loss of generality, we will assume $g(R)=R^{3}$, i.e., $\beta_{0}=0$ and $\beta_{1}=1$ (the extension to other cases is straightforward). Therefore $s_{n}^{(3)}=s_{n} s_{n}^{*} s_{n}$ and these samples can be written as

$$
s_{n}^{(3)}=\frac{1}{\sqrt{N^{3}}} \sum_{k^{(3)} \in \mathcal{K}^{(3)}} S_{k_{1}} S_{k_{2}}^{*} S_{k_{3}} e^{j 2 \pi\left(k_{1}-k_{2}+k_{3}\right) n / N^{\prime}},
$$

with $k^{(3)}=\left(k_{1}, k_{2}, k_{3}\right)$ and $\mathcal{K}^{(3)}=\mathcal{K}^{(1)} \times \mathcal{K}^{(1)} \times \mathcal{K}^{(1)}$. We define two sets $\mathcal{K}_{1}^{(3)}=\left\{\left(k_{1}, k_{2}, k_{3}\right) \in \mathcal{K}^{(3)}: k_{2}=k_{1}\right\}$ and $\mathcal{K}_{2}^{(3)}=\left\{\left(k_{1}, k_{2}, k_{3}\right) \in \mathcal{K}^{(3)}: k_{2}=k_{3}\right\}$ and rewrite samples $s_{n}^{(3)}$ as a sum of two parcels, i.e.,

$$
\begin{aligned}
s_{n}^{(3)} & =\frac{1}{\sqrt{N^{3}}} \sum_{k^{(3)} \in \mathcal{U}^{(3)}} S_{k}\left|S_{k^{\prime}}\right|^{2} e^{j 2 \pi k n / N^{\prime}} \\
& +\frac{1}{\sqrt{N^{3}}} \sum_{k^{(3)} \in \mathcal{K}^{(3)} \backslash \mathcal{U}^{(3)}} S_{k_{1}} S_{k_{2}}^{*} S_{k_{3}} e^{j 2 \pi\left(k_{1}-k_{2}+k_{3}\right) n / N^{\prime}},
\end{aligned}
$$

with $\mathcal{U}^{(3)}=\mathcal{K}_{1}^{(3)} \cup \mathcal{K}_{2}^{(3)}$. Clearly $\left|\mathcal{K}^{(3)}\right|=N^{3},\left|\mathcal{K}_{1}^{(3)}\right|=$ $\left|\mathcal{K}_{2}^{(3)}\right|=N$ and, since $\left|\mathcal{U}^{(3)}\right|=\left|\mathcal{K}_{1}^{(3)} \cup \mathcal{K}_{2}^{(3)}\right|=$ $\left|\mathcal{K}_{1}^{(3)}\right|+\left|\mathcal{K}_{2}^{(3)}\right|-\left|\mathcal{K}_{1}^{(3)} \cap \mathcal{K}_{2}^{(3)}\right|=2 N-1(|S|$ denotes the cardinal of set $S$ ), the first summation in (5) reduces to $(2 N-1) E\left[\left|S_{k}\right|^{2}\right] \sum_{k \in \mathcal{K}^{(1)}} S_{k} e^{j 2 \pi k n / N^{\prime}}$. Hence the two parcels in equation (5) obviously correspond to useful and self-interfering components, i.e., $s_{n}^{(3)}=\alpha^{(3)} s_{n}+d_{n}^{(3)}$, with

$$
\alpha^{(3)}=\frac{2 N-1}{N} E\left[\left|S_{k}\right|^{2}\right]=\left(2-\frac{1}{N}\right) E\left[\left|S_{k}\right|^{2}\right]
$$

and

$$
d_{n}^{(3)}=\frac{1}{\sqrt{N^{3}}} \sum_{k^{(3)} \in \mathcal{K}^{(3)} \backslash \mathcal{U}^{(3)}} S_{k_{1}} S_{k_{2}}^{*} S_{k_{3}} e^{j 2 \pi\left(k_{1}-k_{2}+k_{3}\right) n / N^{\prime}} .
$$

Let us define multiplicity of subcarrier $k$ as the number of times we get $k_{1}-k_{2}+k_{3}-\ldots-k_{2 p}+k_{2 p+1}=k$ for all possible $(2 p+1)$-tuples of set $\mathcal{K}^{(2 p+1)}\left(\mathcal{K}^{(n)}\right.$ denotes the $n$ ary Cartesian product over set $\left.\mathcal{K}^{(1)}\right)$. Letting $M_{k}^{(2 p+1)}$ denote the multiplicity of the $k$ th subcarrier associated to a nonlinear characteristic of type $R^{2 p+1}$ and $M_{k}^{(2 p+1 \rightarrow 2 \gamma+1)}$ denote the multiplicity of that subcarrier associated with the IMP of order $\gamma$, with $\gamma \leq p$, produced by the same nonlinear characteristic, it is clear that

$$
M_{k}^{(1)}= \begin{cases}1, & k=0,1, \ldots, N-1 \\ 0, & \text { otherwise }\end{cases}
$$

and $\sum_{k=0}^{N-1} M_{k}^{(1)}=N$. For the signal $s_{n}^{(3)}$ the multiplicity of subcarrier $k$ is $M_{k}^{(3)}$, where the block $M^{(3)}=\left\{M_{k}^{(3)}, k=\right.$ $-4 N+1,-4 N+2, \ldots, 5 N-2\}$ is the convolution of the augmented blocks $M^{(1)}=\left\{M_{k}^{(1)}, k=-N,-N+1, \ldots, 2 N-\right.$ 1 , i.e., $M^{(3)}=M^{(1)} * M^{(1)} * M^{(1)}$. The augmented block is obtained by adding $2 N$ zeros to the initial block, thus ensuring there is no aliasing when computing the convolution. The block $M^{(3)}$ can be obtained by computing the IDFT of the block $\left\{m_{n}^{(1)} m_{-n}^{(1)} m_{n}^{(1)}, n=-N,-N+1, \ldots, 2 N-1\right\}$, with $\left\{m_{n}^{(1)}, n=-N,-N+1, \ldots, 2 N-1\right\}=$ DFT $\left\{M_{k}^{(1)}, k=\right.$ $-N,-N+1, \ldots, 2 N-1\}$. The generalization of $M^{(2 p+1)}$ to any value of $p$ is straightforward.

The discrete convolution $u=x * y$ is given by

$$
u_{k}=\sum_{k^{\prime}=\mathrm{Max}_{1}}^{\min _{1}} x_{k^{\prime}} y_{k-k^{\prime}+1},
$$

$k=0, \ldots, 2 M-2$, with $\operatorname{Max}_{1}=\max (1, k-M+1)$ and $\min _{1}=\min (k, M)$, where $M$ is the length of vectors $x$ and $y$. Consequently, for $v=u * z=x * y * z$ we have

$$
\begin{aligned}
v_{k} & =\sum_{k^{\prime \prime}=\operatorname{Max}_{2}}^{\min _{2}} u_{k} z_{k-k^{\prime \prime}+1} \\
& =\sum_{k^{\prime \prime}=\operatorname{Max}_{2}}^{\min _{2}} \sum_{k^{\prime}=\operatorname{Max}_{1}}^{\min _{1}} x_{k^{\prime}} y_{k-k^{\prime}+1} z_{k-k^{\prime \prime}+1},
\end{aligned}
$$

$k=0, \ldots, 3 M-3$, with $\operatorname{Max}_{2}=\max (1, k-2 M+2)$ and $\min _{2}=\min (k, M)$. It follows that

$$
M_{k}^{(2)}= \begin{cases}N / 2+k, & -N / 2+1 \leq k \leq N / 2-1 \\ 3 N / 2-k, & N / 2 \leq k \leq 3 N / 2-1 \\ 0, & \text { otherwise }\end{cases}
$$

and $M_{k}^{(3)}$ is given by (12). Obviously $\sum_{k=-N / 2+1}^{3 N / 2-1} M_{k}^{(2)}=$ $N^{2}$ and $\sum_{k=-N+1}^{2 N-2} M_{k}^{(3)}=N^{3}$. The useful component has multiplicity

$$
M_{k}^{(3 \rightarrow 1)}= \begin{cases}2 N-1, & 0 \leq k \leq N-1 \\ 0, & \text { otherwise }\end{cases}
$$

which means that the multiplicity of the self-interference samples is $M_{k}^{(3 \rightarrow 3)}=M_{k}^{(3)}-M_{k}^{(3 \rightarrow 1)}$ and has sum

$$
\begin{aligned}
\sum_{k=-N+1}^{2 N-2} M_{k}^{(3 \rightarrow 3)} & =\sum_{k=-N+1}^{2 N-2} M_{k}^{(3)}-\sum_{k=0}^{N-1} M_{k}^{(3 \rightarrow 1)} \\
& =N^{3}-2 N^{2}+N .
\end{aligned}
$$

The evolution of multiplicities $M_{k}^{(3)}, M_{k}^{(3 \rightarrow 3)}$ and $M_{k}^{(3 \rightarrow 1)}$ are depicted in Fig. 1.A.

The power of the useful component is

$$
\begin{aligned}
S^{(3)}=P_{1}^{(3)} & =\frac{\left(\alpha^{(3)}\right)^{2}}{2} E\left[\left|S_{k}\right|^{2}\right] \\
& =\frac{1}{2}\left(2-\frac{1}{N}\right)^{2}\left(E\left[\left|S_{k}\right|^{2}\right]\right)^{3}
\end{aligned}
$$




$$
M_{k}^{(3)}= \begin{cases}(k+N)(k+N+1) / 2, & -N+1 \leq k \leq-1 \\ 3 N(2 k+N+1) / 2-(k+N)(k+N+1), & 0 \leq k \leq N-1 \\ (2 N-k)(2 N-k-1) / 2, & N \leq k \leq 2 N-2 \\ 0, & \text { otherwise }\end{cases}
$$
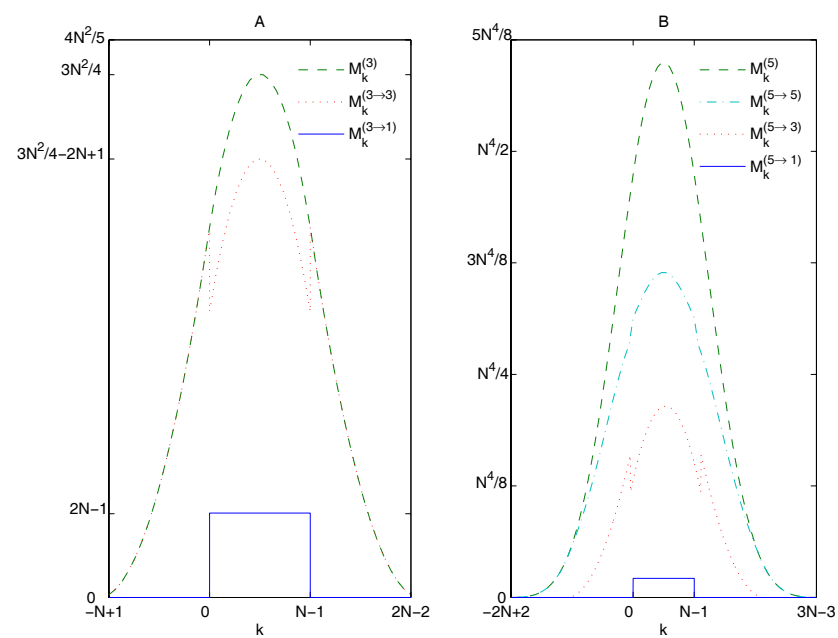

Fig. 1. Evolution of $M_{k}^{(3)}$ (dashed line), $M_{k}^{(3 \rightarrow 3)}$ (dotted line) and $M_{k}^{(3 \rightarrow 1)}$ (solid line) (A) and $M_{k}^{(5)}$ (dashed line), $M_{k}^{(5 \rightarrow 5)}$ (dash-dotted line), $M_{k}^{(5 \rightarrow 3)}$ (dotted line) and $M_{k}^{(5 \rightarrow 1)}$ (solid line) (B).

and, recalling that $d_{n}^{(3)}$ is given by (7), the power of the selfinterference component is

$$
\begin{aligned}
I^{(3)}=P_{3}^{(3)} & =2 ! \frac{N^{3}-2 N^{2}+N}{N^{3}} \frac{\left(E\left[\left|S_{k}\right|^{2}\right]\right)^{3}}{2} \\
& =\left(1-\frac{2}{N}+\frac{1}{N^{2}}\right)\left(E\left[\left|S_{k}\right|^{2}\right]\right)^{3},
\end{aligned}
$$

where the factor 2 ! is the number of times we can get repetitions of the values taken by $k_{1}$ and $k_{3}$.

If $E\left[\left|S_{k}\right|^{2}\right]=2 \sigma^{2}$ then

$$
\begin{aligned}
\alpha^{(3)} & =\left(4-\frac{2}{N}\right) \sigma^{2} \\
P_{1}^{(3)} & =\left(4-\frac{2}{N}\right)^{2} \sigma^{6} \\
P_{3}^{(3)} & =8\left(1-\frac{2}{N}+\frac{1}{N^{2}}\right) \sigma^{6}
\end{aligned}
$$

For a large number of subcarriers, i.e., $N \gg 1$, these expressions can be approximated by $\alpha^{(3)} \approx 4 \sigma^{2}, P_{1}^{(3)} \approx 16 \sigma^{6}$ and $P_{3}^{(3)} \approx 8 \sigma^{6}$. Notice these are the same values we get by using the Gaussian approximation results presented in appendix B with $g(R)=R^{3}$.

\section{B. Nonlinear Characteristic of Order 5}

Let us now consider a bandpass memoryless nonlinear with characteristic of order 5, i.e., $\beta_{m}=0$ for $m \geq 3$. Again, without loss of generality, we will assume $g(R)=R^{5}$, i.e., $\beta_{0}=0, \beta_{1}=0$ and $\beta_{2}=1$. Therefore,

$$
\begin{aligned}
& s_{n}^{(5)}=s_{n} s_{n}^{*} s_{n} s_{n}^{*} s_{n} \\
& =\frac{1}{\sqrt{N^{5}}} \sum_{k^{(5)} \in \mathcal{K}^{(5)}} S_{k_{1}} S_{k_{2}}^{*} S_{k_{3}} S_{k_{4}}^{*} S_{k_{5}} e^{j 2 \pi\left(k_{1}-k_{2}+k_{3}-k_{4}+k_{5}\right) n / N^{\prime}}
\end{aligned}
$$

with $k^{(5)}=\left(k_{1}, k_{2}, k_{3}, k_{4}, k_{5}\right)$ and $\mathcal{K}^{(5)}=\mathcal{K}^{(1)} \times \mathcal{K}^{(1)} \times$ $\mathcal{K}^{(1)} \times \mathcal{K}^{(1)} \times \mathcal{K}^{(1)}$. In appendix $\mathrm{A}$ it is shown that we can write $s_{n}^{(5)}$ as a sum of useful and self-interference components $s_{n}^{(5)}=\alpha^{(5)} s_{n}+d_{n}^{(5)}$, with $\alpha^{(5)}$ given by (33) and selfinterference samples given by (35).

Let $M_{k}^{(5)}$ be the total multiplicity of subcarrier $k, M_{k}^{(5 \rightarrow 1)}$ the multiplicity of the useful component and $M_{k}^{(5 \rightarrow 3)}$ and $M_{k}^{(5 \rightarrow 5)}$ the multiplicities of the self-interference components. In appendix $\mathrm{A}$ it is shown that these multiplicities are given by (32), (36) and (38), respectively. The evolution of $M_{k}^{(5)}$, $M_{k}^{(5 \rightarrow 1)}, M_{k}^{(5 \rightarrow 3)}$ and $M_{k}^{(5 \rightarrow 5)}$ is depicted in Fig. 1.B.

In case $E\left[\left|S_{k}\right|^{2}\right]=2 \sigma^{2}$, using the results of appendix A, we can write the power of the useful and self-interfering components as

$$
\begin{aligned}
\alpha^{(5)} & =4\left(6-\frac{9}{N}+\frac{4}{N^{2}}\right) \sigma^{4} \\
P_{1}^{(5)} & =16\left(6-\frac{9}{N}+\frac{4}{N^{2}}\right)^{2} \sigma^{10} \\
P_{3}^{(5)} & =32\left(6-\frac{9}{N}\right)^{2}\left(1-\frac{2}{N}+\frac{1}{N^{2}}\right) \sigma^{10} \\
P_{5}^{(5)} & =192\left(1-\frac{6}{N}+\frac{15}{N^{2}}-\frac{17}{N^{3}}+\frac{7}{N^{4}}\right) \sigma^{10}
\end{aligned}
$$

and the total power of the self-interference samples is simply $I^{(5)}=P_{3}^{(5)}+P_{5}^{(5)}$. When the number of subcarriers is high the following approximations can be used $\alpha^{(5)} \approx 24 \sigma^{4}$, $P_{1}^{(5)} \approx 576 \sigma^{10}, P_{3}^{(5)} \approx 1152 \sigma^{10}, P_{5}^{(5)} \approx 192 \sigma^{10}$ and $I^{(5)} \approx 1344 \sigma^{10}$. Again we can notice that these are the same expressions we get by using $g(R)=R^{5}$ in the results of appendix B.

\section{Generalization}

Previous results can be generalized for large values of $N$ noticing that the multiplicities for the signal $s_{n}^{(2 p+1)}$ are approximately given by

$$
\begin{aligned}
M_{k}^{(2 p+1 \rightarrow 2 \gamma+1)} & \approx\left(\begin{array}{c}
p \\
p-\gamma
\end{array}\right) P(p+1, p-\gamma) N^{p+\gamma} \\
& =\frac{p !}{\gamma !(p-\gamma) !} \frac{(p+1) !}{(\gamma+1) !} N^{p+\gamma}
\end{aligned}
$$

if $\gamma<p$, with $P(n, r)$ denoting "permutation of $n$ elements $r$ by $r "$, and by

$$
M_{k}^{(2 p+1 \rightarrow 2 p+1)} \approx M_{k}^{(2 p+1)}-\sum_{l=0}^{p-1} M_{k}^{(2 p+1 \rightarrow 2 l+1)} .
$$


TABLE I

COMPARISON OF $\alpha^{2 p+1} / \sigma^{2 p}$ FOR DIFFERENT VALUES OF $N$.

\begin{tabular}{c|ccc|ccc} 
& \multicolumn{3}{|c|}{$\alpha^{(3)}$} & & $\alpha^{(5)}$ & \\
$N$ & approx & exact & simul & approx & exact & simul \\
\hline 16 & 4 & 3.875 & 3.873 & 24 & 21.813 & 21.851 \\
32 & 4 & 3.938 & 3.937 & 24 & 22.891 & 22.967 \\
64 & 4 & 3.969 & 3.966 & 24 & 23.441 & 23.437 \\
128 & 4 & 3.984 & 3.986 & 24 & 23.720 & 23.713 \\
256 & 4 & 3.992 & 3.991 & 24 & 23.860 & 23.867
\end{tabular}

if $\gamma=p$, where $\sum_{k} M_{k}^{(2 p+1)}=N^{2 p+1}$.

Thus the power of the useful and the self-interfering components can be approximated by

$$
\begin{aligned}
& P_{2 \gamma+1}^{(2 p+1)} \approx \frac{1}{N^{2 p+1}} \gamma !(\gamma+1) !\left(\sum_{k} M_{k}^{(2 p+1 \rightarrow 2 \gamma+1)}\right)^{2} \\
& \cdot \frac{\left(E\left[\left|S_{k}\right|^{2}\right]\right)^{2 p+1}}{2 N^{2 \gamma+1}} \\
&=\frac{1}{2} \frac{1}{\gamma !(\gamma+1) !}\left(\frac{p !(p+1) !}{(p-\gamma) !}\right)^{2}\left(E\left[\left|S_{k}\right|^{2}\right]\right)^{2 p+1}
\end{aligned}
$$

for $\gamma<p$ and, if $p=\gamma$ by

$$
\begin{aligned}
P_{2 p+1}^{(2 p+1)} & \approx \frac{1}{N^{2 p+1}} p !(p+1) !\left(N^{2 p+1}\right)^{2} \frac{\left(E\left[\left|S_{k}\right|^{2}\right]\right)^{2 p+1}}{2 N^{2 p+1}} \\
& \approx \frac{p !(p+1) !}{2}\left(E\left[\left|S_{k}\right|^{2}\right]\right)^{2 p+1} .
\end{aligned}
$$

If $E\left[\left|S_{k}\right|^{2}\right]=2 \sigma^{2}$ we get

$$
P_{2 \gamma+1}^{(2 p+1)} \approx \frac{1}{\gamma !(\gamma+1) !}\left(\frac{p !(p+1) !}{(p-\gamma) !}\right)^{2} 2^{2 p} \sigma^{4 p+2}
$$

and $P_{2 p+1}^{(2 p+1)} \approx p !(p+1) ! 2^{2 p} \sigma^{4 p+2}$, which are the expressions obtained by using the Gaussian approximation (see appendix B).

\section{ACCURACY OF THE GAUSSIAN APPROXIMATION}

From the comparison of exact results and the ones obtained using the Gaussian approximation it is clear that the Gaussian approximation leads to errors. In this section we present some results concerning these approaches.

The Gaussian approximation leads to errors of $2 / N$ and $36 / N-16 / N^{2}$ on the evaluation of $\alpha^{(3)}$ and $\alpha^{(5)}$ values, respectively. Table I shows values obtained using the exact characterization, the Gaussian approximation and simulation for $\alpha^{(3)}$ and $\alpha^{(5)}$ and several values of $N$. It can be seen that values obtained using the exact expressions are very close to the ones obtained by simulation and that for values of $N$ greater then 100 there are no significant differences between the exact and the approximated values.

As mentioned before, when the number of subcarriers is high it is usual to consider $D_{k}$ exhibits quasi-Gaussian characteristics for any $k$. This behavior of the nonlinear distortion component at the subcarrier level is the result of having a large number of contributions at each subcarrier, i.e., a large multiplicity. For $g(R)=R^{3}$, the number of contributions on subcarrier $k$ is $M_{k}^{(3 \rightarrow 3)}=M_{k}^{(3)}-M_{k}^{(3 \rightarrow 1)}$, which has a quadratic characteristic with a maximum of order $N^{2}$. Due to this maximum in the cubic nonlinearity case (and more if

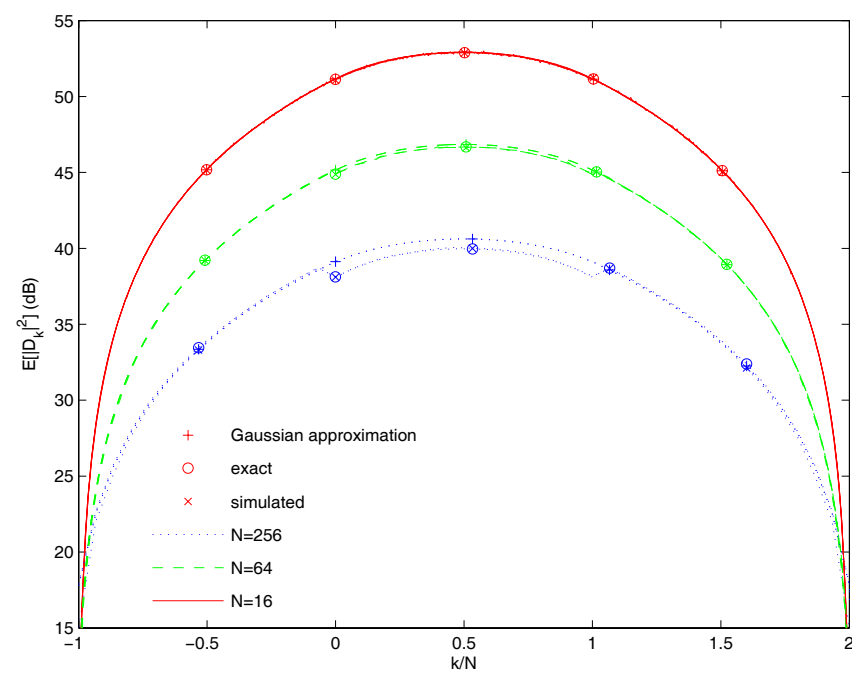

Fig. 2. Comparison of Gaussian approximation, exact and simulated values for $E\left[\left|D_{k}\right|^{2}\right]$ and $g(R)=R^{3}$.

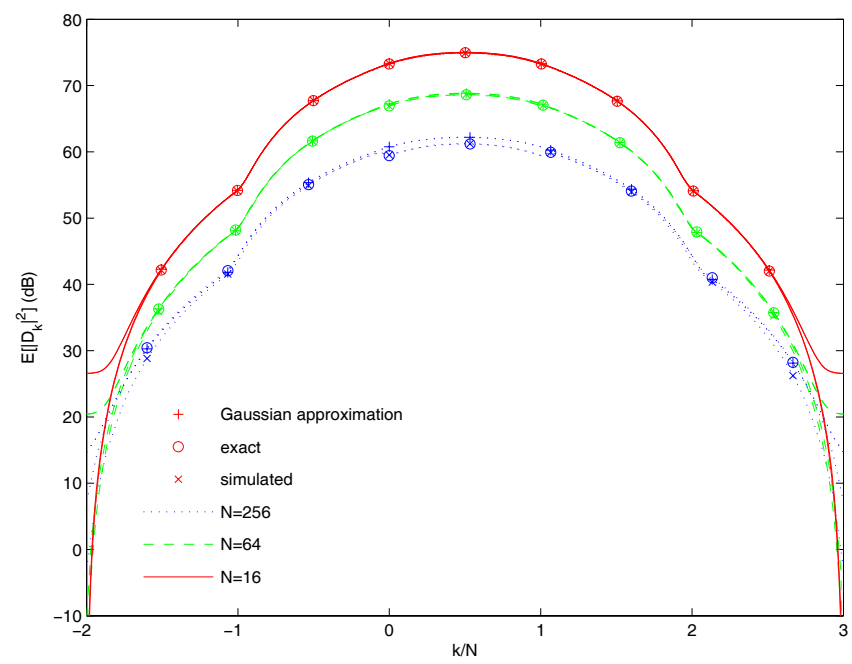

Fig. 3. Comparison of Gaussian approximation, exact and simulated values for $E\left[\left|D_{k}\right|^{2}\right]$ and $g(R)=R^{5}$.

we have higher order terms), the Gaussian approximation is accurate whenever $N^{2} \gg 1$ (say $N>10$ ). This means that for $N=16$ subcarriers we already have an almost Gaussian behavior at the subcarrier level, at least for the central part of the spectrum. However, for the subcarriers at the edge of the band the multiplicity starts with small values. In fact, for a cubic nonlinearity the number of contributions grows with $k^{2}$ but is very low at the edges of the band (see (12) and Fig. 1.A). The same happens with $g(R)=R^{5}$, since the number of contributions on subcarrier $k$ is $M_{k}^{(5)}-M_{k}^{(5 \rightarrow 1)}$ (see (32), (36) and Fig. 1.B), which depends on $M_{k}^{(3 \rightarrow 3)}$. This implies the Gaussian approximation is not accurate at the edges of the spectrum. A comparison of $E\left[\left|D_{k}\right|^{2}\right]$ values obtained using the Gaussian approximation, the exact characterization and simulations is shown in Figs. 2 and 3.

As an example let us now consider a Rapp characteristic, which can be used to model a Solid State Power Amplifier (SSPA) [10] and includes an ideal clipping as a particular 


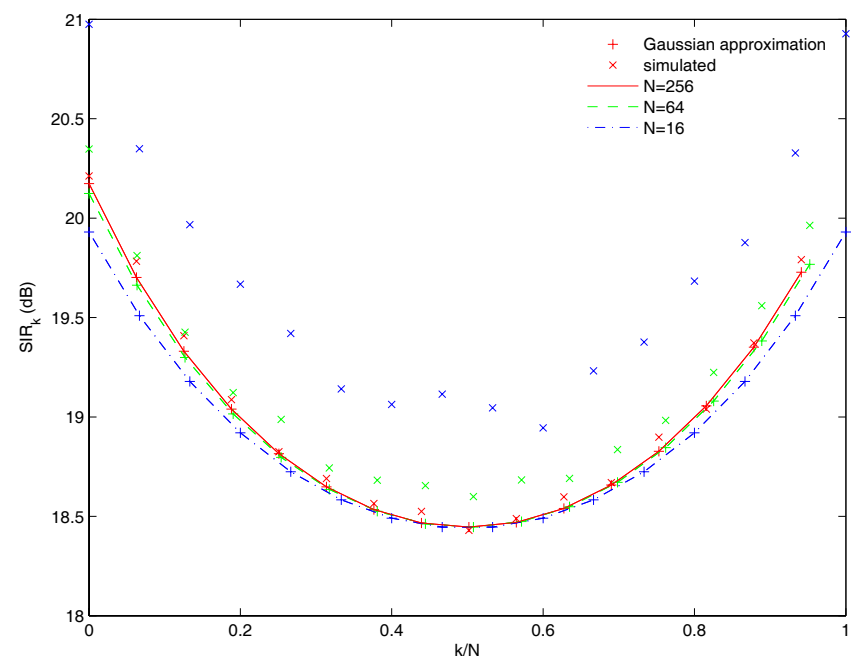

Fig. 4. Comparison of Gaussian approximation and simulated values for $\operatorname{SIR}_{k}$ for a SSPA with $q=2$ and $s_{M} / \sigma=2.0$.

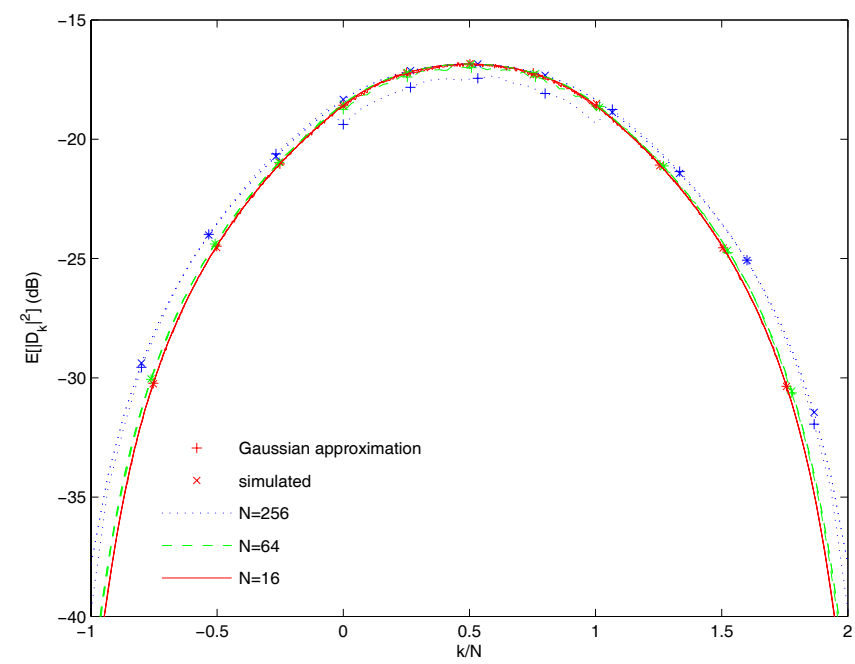

Fig. 5. Comparison of Gaussian approximation and simulated values for $E\left[\left|D_{k}\right|^{2}\right]$ for a SSPA with $q=2$ and $s_{M} / \sigma=2.0$.

case. In this case, $g(R)=R / \sqrt[2 q]{1+\left(R / s_{M}\right)^{2 q}}$, where $s_{M}$ is the saturating amplitude and the parameter $q$ is an integer that controls the smooth transition from linear region to saturation region. Note that, as $q$ grows larger (for $q=1$ this corresponds to an ideal clipping). Figs. 4 and 5 show SIR levels and $E\left[\left|D_{k}\right|^{2}\right]$ values obtained using the Gaussian approximation and simulations for an SSPA with $q=2$ and $s_{M} / \sigma=2.0$. Once again, the values obtained employing the Gaussian approximation are not accurate when we have a small number of subcarriers and our theoretical values are very close to the simulated ones, showing the relevance of our method.

\section{CONCLUSIONS}

In this paper we studied the accuracy of using the Gaussian approach to evaluate the impact of memoryless nonlinear devices in OFDM signals. It is shown that even for a reduced number of subcarriers a multicarrier signal submitted to a nonlinear device can be decomposed in useful and self- interference components. In the cases of nonlinear characteristics of order 3 and 5, exact expressions for the power of these components are derived and compared with the ones obtained using the Gaussian approximation approach. From the presented analysis we concluded that the Gaussian approximation is very good for large values of $N$, as expected. Values found for $\alpha$ are also very precise. However, the PSD of the self-interference component is over-estimated especially in the in-band region, leading to slightly pessimistic SIR levels, with an error inversely proportional to $N$. With respect to the Gaussian approximation of the self-interference component at the subcarrier level, it is very accurate whenever $N^{2}$ is high, except at the edge of the band.

\section{APPENDIX A}

\section{EXACT CHARACTERIZATION OF BANDPASS MEMORYLESS NONLINEARITIES WITH CHARACTERISTIC $g(R)=R^{5}$}

In this appendix we present the exact characterization for a bandpass memoryless nonlinear with characteristic $g(R)=$ $R^{5}$, i.e., with samples at the output of the nonlinear device $s_{n}^{(5)}=s_{n} s_{n}^{*} s_{n} s_{n}^{*} s_{n}$ given by (20).

Having in mind determining the multiplicity of each subcarrier, we define the sets $\mathcal{K}_{m}^{(5)}=\left\{\left(k_{1}, k_{2}, k_{3}, k_{4}, k_{5}\right) \in \mathcal{K}^{(5)}\right.$ : $\left.k_{x}=k_{y} \wedge k_{w}=k_{z}\right\}$, for all $x, w \in\{2,4\}$ and $y, z \in\{1,3,5\}$. Clearly $\left|\mathcal{K}^{(5)}\right|=N^{5}$ and $\left|\mathcal{K}_{m}^{(5)}\right|=N^{2}, m=1, \ldots, 6$. The multiplicity of the useful component is

$$
M_{k}^{(5 \rightarrow 1)}= \begin{cases}\left|\mathcal{U}^{(5)}\right|, & 0 \leq k \leq N-1 \\ 0, & \text { otherwise }\end{cases}
$$

with $\mathcal{U}^{(5)}=\bigcup_{m=1}^{6} \mathcal{K}_{m}^{(5)}$. The cardinal of the set $\mathcal{U}^{(5)}$ can be obtained from

$$
\begin{aligned}
\left|\bigcup_{m=1}^{6} \mathcal{K}_{m}^{(5)}\right| & =\sum_{m=1}^{6}\left|\mathcal{K}_{m}^{(5)}\right|+\sum_{m=2}^{6}(-1)^{m-1} \mathcal{I}_{m}^{(5)} \\
& =\sum_{m=1}^{6}(-1)^{m-1} \mathcal{I}_{m}^{(5)}
\end{aligned}
$$

with $\mathcal{I}_{m}^{(5)}=\sum_{\left\{i_{1}, \ldots, i_{m}\right\} \in \mathcal{C}_{m}^{(5)}}\left|\bigcap_{l=1}^{m} \mathcal{K}_{i_{l}}^{(5)}\right|$, where $\mathcal{C}_{m}^{(5)}$ represents is the set of all subsets of $\{1,2, \ldots, 6\}$ that contain $m$ elements. Clearly $\left|\mathcal{C}_{m}^{(5)}\right|=\left(\begin{array}{c}6 \\ m\end{array}\right)$ hence for $m=2$ the number of subsets is $\left(\begin{array}{l}6 \\ 2\end{array}\right)=15$. It can be shown that of these 9 have cardinal $N$ and the remaining have cardinal 1, which means that $\mathcal{I}_{2}^{(5)}=9 N+6$. For $m=3,4,5$ and 6 all subsets have cardinal 1 , hence $\mathcal{I}_{m}^{(5)}=\left(\begin{array}{c}6 \\ m\end{array}\right)$ and we can write $\left|\mathcal{U}^{(5)}\right|=\mathcal{I}_{1}^{(5)}-\mathcal{I}_{2}^{(5)}+\mathcal{I}_{3}^{(5)}-\mathcal{I}_{4}^{(5)}+\mathcal{I}_{5}^{(5)}-\mathcal{I}_{6}^{(5)}=6 N^{2}-9 N+4$, which means

$$
M_{k}^{(5 \rightarrow 1)}=\left(6 N^{2}-9 N+4\right) M_{k}^{(1)}
$$

and the sum of the multiplicities of the useful and selfinterference samples are, respectively, $\sum_{k=0}^{N-1} M_{k}^{(5 \rightarrow 1)}=$ $6 N^{3}-9 N^{2}+4 N$ and $\sum_{k=-2 N+2}^{3 N-3} M_{k}^{(5)}-\sum_{k=0}^{N-1} M_{k}^{(5 \rightarrow 1)}=$ $N^{5}-6 N^{3}+9 N^{2}-4 N$. 
Thus we can write $s_{n}^{(5)}$ as a sum of a useful and a self- and interference components $s_{n}^{(5)}=\alpha^{(5)} s_{n}+d_{n}^{(5)}$, with

$$
\begin{aligned}
\alpha^{(5)} & =\frac{6 N^{2}-9 N+4}{N^{2}}\left(E\left[\left|S_{k}\right|^{2}\right]\right)^{2} \\
& =\left(6-\frac{9}{N}+\frac{4}{N^{2}}\right)\left(E\left[\left|S_{k}\right|^{2}\right]\right)^{2}
\end{aligned}
$$

and

$$
\begin{aligned}
d_{n}^{(5)}=\frac{1}{\sqrt{N^{5}}} \sum_{k^{(5)} \in \mathcal{K}^{(5)} \backslash \mathcal{U}^{(5)}} S_{k_{1}} S_{k_{2}}^{*} S_{k_{3}} S_{k_{4}}^{*} S_{k_{5}} \\
\cdot e^{j 2 \pi\left(k_{1}-k_{2}+k_{3}-k_{4}+k_{5}\right) n / N^{\prime}} .
\end{aligned}
$$

The self-interference samples can be written as

$$
\begin{aligned}
& d_{n}^{(5)}= \frac{1}{N} \sum_{k} M_{k}^{(5 \rightarrow 3)} E\left[\left|S_{k}\right|^{2}\right] \\
& \underbrace{\frac{1}{\sqrt{N^{3}}} \sum_{k^{\prime}} \sum_{k^{\prime \prime}} \sum_{k^{\prime \prime \prime}} S_{k^{\prime}} S_{k^{\prime \prime}}^{*} S_{k^{\prime \prime \prime}} e^{j 2 \pi\left(k^{\prime}-k^{\prime \prime}+k^{\prime \prime \prime}\right) n / N^{\prime}}}_{\approx d_{n}^{(3)}} \\
&+ \frac{1}{\sqrt{N^{5}}} \sum_{k_{1}} \sum_{k_{2}} \sum_{k_{3}} \sum_{k_{4}} \sum_{k_{5}} S_{k_{1}} S_{k_{2}}^{*} S_{k_{3}} S_{k_{4}}^{*} S_{k_{5}} \\
& \cdot e^{j 2 \pi\left(k_{1}-k_{2}+k_{3}-k_{4}+k_{5}\right) n / N^{\prime}} .
\end{aligned}
$$

It can be shown that

$$
M_{k}^{(5 \rightarrow 3)} \approx(6 N-9) M_{k}^{(3 \rightarrow 3)}+(N-2) M_{k}^{(1)}
$$

and

$$
\sum_{k=-2 N+2}^{3 N-3} M_{k}^{(5 \rightarrow 3)}=6 N^{4}-21 N^{3}+26 N^{2}-11 N .
$$

Obviously

$$
M_{k}^{(5 \rightarrow 5)} \approx M_{k}^{(5)}-M_{k}^{(5 \rightarrow 3)}-M_{k}^{(5 \rightarrow 1)}
$$

and

$$
\begin{aligned}
& \sum_{k=-2 N+2}^{3 N-3} M_{k}^{(5 \rightarrow 5)}=\sum_{k=-2 N+2}^{3 N-3} M_{k}^{(5)}-\sum_{k=-2 N+2}^{3 N-3} M_{k}^{(5 \rightarrow 3)} \\
& \quad-\sum_{k=0}^{N-1} M_{k}^{(5 \rightarrow 1)}=N^{5}-6 N^{4}+15 N^{3}-17 N^{2}+7 N .
\end{aligned}
$$

The evolution of $M_{k}^{(5)}, M_{k}^{(5 \rightarrow 1)}, M_{k}^{(5 \rightarrow 3)}$ and $M_{k}^{(5 \rightarrow 5)}$ is depicted in Fig. 1.B.

The power of the useful component is

$$
S^{(5)}=P_{1}^{(5)}=\frac{\left(\alpha^{(5)}\right)^{2}}{2} E\left[\left|S_{k}\right|^{2}\right]
$$

and the power of the self-interfering components are

$$
\begin{aligned}
P_{3}^{(5)} & =\frac{1}{N^{2}}(6 N-9)^{2}\left(E\left[\left|S_{k}\right|^{2}\right]\right)^{2} P_{3}^{(3)} \\
& =\left(6-\frac{9}{N}\right)^{2}\left(1-\frac{2}{N}+\frac{1}{N^{2}}\right)\left(E\left[\left|S_{k}\right|^{2}\right]\right)^{5}
\end{aligned}
$$

$$
P_{5}^{(5)}=6\left(1-\frac{6}{N}+\frac{15}{N^{2}}-\frac{17}{N^{3}}+\frac{7}{N^{4}}\right)\left(E\left[\left|S_{k}\right|^{2}\right]\right)^{5} .
$$

Clearly the total power of the self-interference samples is $I^{(5)}=P_{3}^{(5)}+P_{5}^{(5)}$.

\section{APPENDIX B}

\section{ANALYTICAL CHARACTERIZATION OF NONLINEARLY Distorted Multicarrier Signals USING THE GAUSSIAN APPROXIMATION}

In this appendix we particularize the results obtained using the Gaussian approximation (see [5]) for the case where $g(R)=R^{2 p+1}$. In this case $\alpha$ can be obtained from

$$
\begin{aligned}
& \alpha^{(2 p+1)}=\frac{1}{2 \sigma^{4}} \int_{0}^{+\infty} R^{2} R^{2 p+1} e^{-\frac{R^{2}}{2 \sigma^{2}}} d R \\
& =\frac{1}{2} \sigma^{2 p}(2 p+2)(2 p)(2 p-2) \ldots 2=2^{p}(p+1) ! \sigma^{2 p},
\end{aligned}
$$

and the average power of the signal at the nonlinearity output is given by

$$
\begin{aligned}
& P_{\text {out }}^{(2 p+1)}=\frac{1}{2 \sigma^{2}} \int_{0}^{+\infty} R R^{4 p+2} e^{-\frac{R^{2}}{2 \sigma^{2}}} d R \\
& \quad=\frac{1}{2} \sigma^{4 p+2}(4 p+2)(4 p) \ldots 2=2^{2 p}(2 p+1) ! \sigma^{4 p+2} .
\end{aligned}
$$

The intermodulation products can be obtained from

$$
P_{2 \gamma+1}^{(2 p+1)}=\frac{1}{\gamma !(\gamma+1) !}\left(\frac{p !(p+1) !}{(p-\gamma) !}\right)^{2} 2^{2 p} \sigma^{4 p+2} .
$$

In the particular case of $\gamma=p$ we simply get $P_{2 p+1}^{(2 p+1)}=$ $p !(p+1) ! 2^{2 p} \sigma^{4 p+2}$.

\section{REFERENCES}

[1] R. Gross and D. Veeneman, "SNR and spectral properties for a clipped DMT ADSL signal," in Proc. 1994 IEEE International Conf. Commun., vol. 2, pp. 843-847.

[2] A. Chini, Y. Wu, M. El-Tanany, and S. Mahmoud, "Hardware nonlinearities in digital TV broadcasting using OFDM modulation," IEEE Trans. Broadcast., vol. 44, no. 1, pp. 12-21, Mar. 1998.

[3] P. Banelli and S. Cacopardi, "Theoretical analysis and performance of OFDM signals in nonlinear AWGN channels," IEEE Trans. Commun., vol. 48, no. 3, pp. 430-441, Mar. 2000.

[4] D. Dardari, V. Tralli, and A. Vaccari, "A theoretical characterization of nonlinear distortion effects in OFDM systems," IEEE Trans. Commun., vol. 48, no. 10, pp. 1755-1764, Oct. 2000.

[5] R. Dinis and A. Gusmão, "A class of nonlinear signal-processing schemes for bandwidth-efficient OFDM transmission with low envelope fluctuation," IEEE Trans. Commun., vol. 52, no. 11, pp. 2009-2018, Nov. 2004.

[6] R. Price, "A useful theorem for nonlinear devices having Gaussian inputs," IRE Trans. Inf. Theory, vol. 4, no. 2, pp. 69-72, June 1958.

[7] G. Stette, "Calculation of intermodulation from a single carrier amplitude characteristic," IEEE Trans. Commun., vol. 22, no. 3, pp. 319-323, Mar. 1974.

[8] H. Nikopour and S. Jamali, "On the performance of OFDM systems over a Cartesian clipping channel: a theoretical approach," IEEE Trans. Wireless Commun., vol. 3, no. 6, pp. 2083-2096, Nov. 2004.

[9] A. Saleh, "Frequency-independent and frequency-dependent nonlinear models of TWT amplifiers," IEEE Trans. Commun., vol. 29, no. 11, pp. 1715-1720, Nov. 1981.

[10] C. Rapp, "Effects of HPA-nonlinearity on a 4-DPSK/OFDM-signal for a digital sound broadcasting system," in Proc. 1991 European Conf. Satellite Commun., pp. 179-184. 